\title{
Clinical experience with pamidronate in the treatment of Paget's disease of bone
}

Stephen J Gallacher, Brendan F Boyce, Uday Patel, Andrew Jenkins, Stuart H Ralston, Iain T Boyle

\begin{abstract}
Bisphosphonates have been shown to be effective in treating the increased bone turnover associated with Paget's disease of bone. In this study two groups of patients were treated with pamidronate by intravenous infusion. In group $1(n=15) 30 \mathrm{mg}$ of pamidronate was given once a week for six weeks. A subgroup (group 1A, $n=6$ ) of more severely affected patients (pretreatment serum alkaline phosphatase (ALP) $>1000 \mathrm{U} / 1$, normal range 80-280 U/1) received a further $60 \mathrm{mg}$ weekly for three weeks. Group $2(n=24)$ received 45 $\mathrm{mg}$ of pamidronate every three months for one year.
\end{abstract}

In both groups the level of ALP in serum samples decreased steadily throughout the year. In group 1 the level decreased to a mean value of $230 \mathrm{U} / 1$ ( $95 \%$ confidence interval 188-281) and in group 2 to 297 U/l (227-389). Four of the six patients in group $1 \mathrm{~A}$ achieved normal ALP, whereas ALP remained at an increased level in all of the 10 patients in group 2 whose pretreatment ALP was $>1000$ $U / 1$, suggesting that a dose-response effect exists. The lowest hydroxyproline to creatinine ratios (normal ratio $<0.033$ ) were observed at the end of treatment in group 1 , with a mean ratio of 0.022 (range $0.015-0.033$ ) and at three months after the start of treatment in group 2 with a mean ratio of 0.029 (range $0.022-0.037$ ). There was a significant decrease in the turnover of bone, as measured by whole body retention of radiolabelled bisphosphonate, from a mean of 49.3 to $41 \cdot 0 \%(p<0.01)$.

These data confirm that pamidronate is effective in the management of Paget's disease of bone. For patients with levels of ALP in serum samples of up to four times above the upper limit of the normal reference range, an effective and convenient regimen is $45 \mathrm{mg}$ every three months for one year. For patients with higher levels of ALP higher doses may be more effective.

Bisphosphonates are analogues of pyrophosphate which inhibit the growth ${ }^{1}$ and dissolution ${ }^{2}$ of hydroxyapatite crystals in vitro and inhibit osteoclastic bone resorption in vivo. ${ }^{2}$

These drugs are effective forms of treatment when bone resorption is increased, such as in Paget's disease of bone ${ }^{3}$ and the hypercalcaemias associated with malignancy, ${ }^{4}$ immobilisation, ${ }^{5}$ and thyrotoxicosis. ${ }^{6}$ Other inhibitors of bone resportion such as calcitonin ${ }^{7}$ and mithramycin ${ }^{8}$ are also effective in treating Paget's disease. Significant problems exist, however, with respect to the development of resistance to calcitonin 9 and the toxicity of mithramycin. ${ }^{10}$

Of the bisphosphonates, etidronate (ethane hydroxy bisphosphonate) has been used most widely, ${ }^{11}$ although its use in Paget's disease has been associated with the development of focal osteomalacia even at low doses. ${ }^{12}$ Significant experience has also been obtained with clodronate (dichloromethylene bisphosphonate), which has been shown to be a powerful antiosteoclast agent in Paget's disease. ${ }^{13-15}$ Recent work has suggested that pamidronate (aminohydroxypropylidene bisphosphonate) is both safe and effective in managing Paget's disease ${ }^{16}$; however, optimum dose regimens have yet to be defined. Recent interest has concentrated on the intravenous infusion of pamidronate owing to the variable absorption and gastrointestinal problems associated with doses given by mouth. ${ }^{17}$ It is not yet established whether a dose-response effect exists with pamidronate in Paget's disease similar to that described in the treatment of hypercalcaemia of malignancy. ${ }^{18}$ The aim of this study was to investigate whether a prolonged interval between doses, in this instance three months, is as effective as the more intensive treatment used by other workers, ${ }^{19-21}$ and also to establish whether a dose-response effect exists.

\section{Patients and methods}

Patients with symptomatic Paget's disease were chosen for this study. The diagnosis of Paget's disease was based on finding characteristic abnormalities in biochemistry, radiology, and bone scanning with radionuclides. Where possible, further confirmation was obtained by transiliac bone biopsy. A total of 39 patients was included (table) and the study consisted of two main treatment regimens.

Fifteen patients (group 1) received weekly infusions of $30 \mathrm{mg}$ pamidronate for six weeks. Severe Paget's disease was considered to be represented by a pretreatment alkaline phosphatase (ALP) level in serum samples of $>1000$ U/l (normal range 80-280 U/l). Six patients had ALP levels $>1000 \mathrm{U} / \mathrm{l}$ and these six comprised a subgroup (group 1A) that received infusions of $60 \mathrm{mg}$ per week for three additional weeks (table). Group 2 consisted of 24 patients who received infusions of $45 \mathrm{mg}$ pamidronate every three months for one year, irrespective of their serum alkaline phosphatase levels. The total dose of pamidronate given was the same in both groups, i.e. $180 \mathrm{mg}$, although group $1 \mathrm{~A}$ received a total dose of $360 \mathrm{mg}$. All infusions were given in $500 \mathrm{ml} 0.9 \%$ saline and infused intravenously 
Table Profile of patients before treatment

\begin{tabular}{llll}
\hline & $\begin{array}{l}\text { Group } 1 \\
(n=15)\end{array}$ & $\begin{array}{l}\text { Group } 1 A \\
(n=6)\end{array}$ & $\begin{array}{l}\text { Group 2 } \\
(n=24)\end{array}$ \\
\hline $\begin{array}{l}\text { Sex (M/F)* } \\
\text { Mean age (years) }\end{array}$ & $7 / 8$ & $3 / 3$ & $10 / 14$ \\
Age range (years) & 70 & 67 & 66 \\
Mean ALP (U/1) & $41-87$ & $41-75$ & $56-85$ \\
$\begin{array}{l}\text { 95\% confidence interval } \\
\text { Mean number of lesions on scan }\end{array}$ & 770 & 1725 & 880 \\
$\begin{array}{l}\text { Range of number of lesions } \\
\text { Mean whole body retention of radiolabelled } \\
\text { bisphosphonate (\%) }\end{array}$ & $210-5700$ & $950-3730$ & $175-5600$ \\
95\% confidence interval & $2-10$ & 6 & 4 \\
\hline
\end{tabular}

${ }^{*} \mathrm{M} / \mathrm{F}=$ male to female ratio.

over four hours. Five patients in group 1 and two patients in group 2 had previously received etidronate by mouth. None had received treatment in the year before this study and all were considered to have relapsed biochemically.

Symptomatic responses were assessed by visual analogue scores in 12 patients in group 1 . A random group of six of these patients was given placebo infusions of $0.9 \%$ saline weekly for three weeks at the start of treatment in a single-blind study. These six patients then received pamidronate as described above.

Biochemical variables were measured in group 1 patients after one month of treatment, at the end of treatment, at six months, and at one year. In group 2 patients measurements were made at intervals of three months throughout the study. Serum ALP activity was measured by an autoanalyser (Technicon, Tarrytown, USA), urinary hydroxyproline by resin catalysed hydrolysis, ${ }^{22}$ followed by autoanalyser quantitation, and urinary creatinine by an autoanalyser (Technicon). Urine samples were collected as second voided morning samples after fasting and the results expressed as hydroxyproline to creatinine ratios (normal ratio $<0.033$ ).

Bone turnover was also determined before treatment and after one year by the 24 hour whole body retention of methylene bisphosphonate labelled with technetium-99m. ${ }^{23}$

Every patient gave verbal informed consent before beginning treatment and this study was approved by the local hospital ethical committee.

As the data were skewed, they were logarithmically transformed and a Student's $t$ test was used to assess changes within the group. One way analysis of variance was used to assess changes between different treatment groups. Correlations were carried out using Spearman rank coefficients.

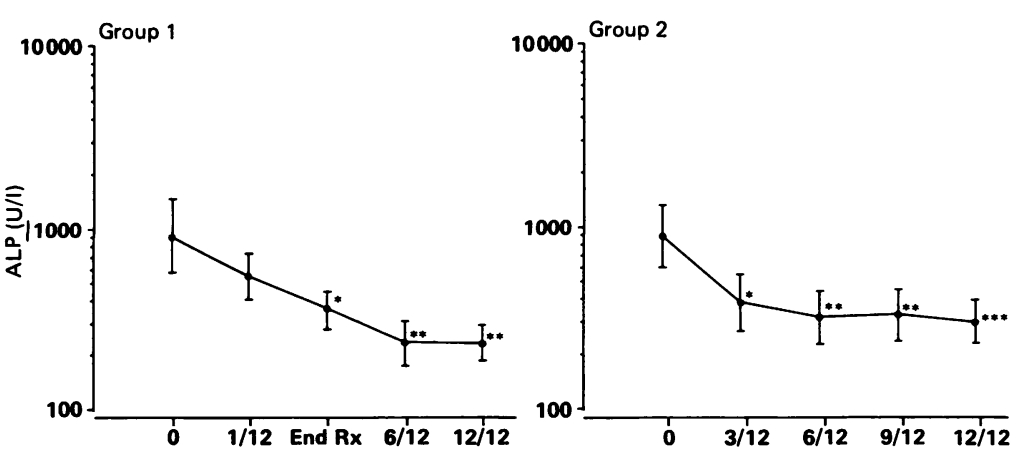

Figure 1 Changes in levels of alkaline phosphatase $(A L P)$ in serum samples. Values shown are means with $95 \%$ confidence intervals on a log scale. Group 1 received $30 \mathrm{mg}$ of pamidronate weekly for six weeks, followed by $60 \mathrm{mg}$ weekly for three weeks if the pretreatment $A L P$ level was $>1000 \mathrm{U} / \mathrm{l}$. Group 2 received $45 \mathrm{mg}$ pamidronate every three months for one year. ${ }^{*} p<0.002,{ }^{*} p<0.0001,{ }^{* * *} p<0.00001$ (versus pretreatment values).
Results

The levels of ALP in serum samples and ratio of hydroxyproline to creatinine in urine throughout the year are expressed as means with $95 \%$ confidence intervals.

ALP levels in serum samples (fig 1) decreased steadily in the two groups throughout the year. At the end of one year the level of ALP was 230 $\mathrm{U} / \mathrm{l}$ in group $1(95 \%$ confidence interval $188-281$, $\mathrm{p}<0.00001)$ and $297 \mathrm{U} / \mathrm{l}(227-389, \mathrm{p}<0.00001)$ in group 2. Fig 2 shows the decrease in ALP level in both groups as absolute values. In group 1 the ALP level in most patients decreased to within or just above the normal range, irrespective of the initial level of ALP. In group 2, however, although there was a significant decrease in ALP levels, this was less marked in the more severely affected patients (i.e. those with a pretreatment ALP of $>1000 \mathrm{U} / \mathrm{l})$. In group 1A four of the six patients with a pretreatment ALP of $>1000 \mathrm{U} / 1$ achieved normal levels of ALP whereas in the remaining two the ALP level decreased to less than 500 U/l. In all nine of the remaining patients in group 1, the ALP decreased to within the normal reference range. In group 2, 10 patients had an ALP level greater than $1000 \mathrm{U} / 1$. None of these patients achieved normal levels of ALP. Of the remaining 13 patients in this group, all but one patient achieved a normal ALP level and in this patient the level was just above the upper limit of the reference range at $310 \mathrm{U} / \mathrm{l}$. The table shows the pretreatment values for these patients.

The maximum decrease in the hydroxyproline to creatinine ratio (fig 3 ) occurred at the end of treatment in group 1 , with a mean of $0.022 \mathrm{U} / 1$ $(0.015-0.033, p<0.001)$, and at three months after the start of treatment in group 2 , with a mean of $0.029 \mathrm{U} / \mathrm{l}(0.022-0.037, \mathrm{p}<0.003)$. In two patients in group 1 and five patients in group 2 , the hydroxyproline to creatinine ratio remained raised.

There was a significant positive correlation between ALP level and the hydroxyproline to creatinine ratio in urine before $(r=0.63$, $\mathrm{p}=0.0002)$ and after treatment $(r=0.41$, $p=0.01$ ). One way analysis of variance gave no statistically significant difference between groups 1 and 2 .

A significant overall improvement in symptoms, assessed by visual analogue pain scores, was seen during treatment. Where placebo infusions were given there was little change in pain scores, although these did subsequently improve (after three weeks) when the patients received the active drug.

There was a general decrease in tracer uptake in radionuclide bone scans after treatment, although this was not always seen. In some patients the tracer uptake was increased in some areas and, in the same patient, decreased in others.

There was a significant decrease in the whole body retention of radiolabelled bisphosphonate at 24 hours over the year of follow up from a mean of $49 \cdot 3$ to $41 \cdot 0 \%(p<0.01)$.

Treatment was well tolerated by most patients, with asymptomatic pyrexia occurring in only $19 \%$. This is lower than expected, 
Group 1 (Weekly treatment)

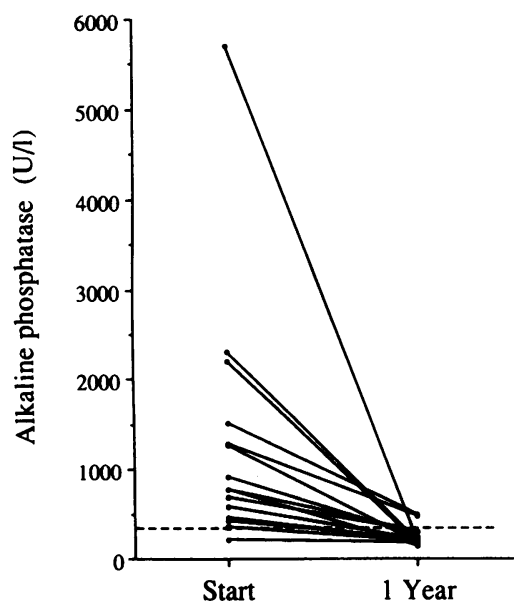

Group 2 (Three monthly treatment)

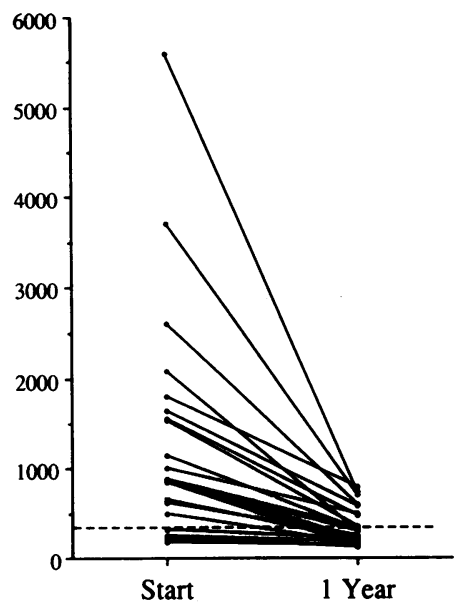

Figure 2 Changes in levels of alkaline phosphatase in serum samples (actual values). Group 1 received $30 \mathrm{mg}$ of pamidronate weekly for six weeks, followed by $60 \mathrm{mg}$ weekly for three weeks if the pretreatment ALP level was $>1000$ U/l. Group 2 received $45 \mathrm{mg}$ every three months for one year.
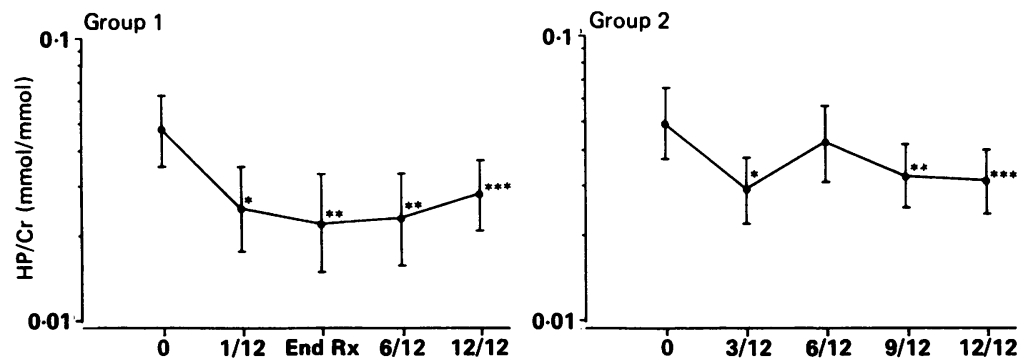

Figure 3 Changes in urinary hydroxyproline to creatinine ratio $(\mathrm{HP} / \mathrm{Cr})$. Values shown are means with $95 \%$ confidence intervals on a log scale. Group 1 received $30 \mathrm{mg}$ of pamidronate weekly for six weeks, followed by $60 \mathrm{mg}$ weekly for three weeks if the pretreatment alkaline phosphatase level was $>1000$ U/l. Group 2 received $45 \mathrm{mg}$ every three months for one year. ${ }^{*} p<0.003,{ }^{* *} p<0.001,{ }^{* * *} p<0.01$ (versus pretreatment values).

perhaps because the first 15 patients treated received pamidronate as outpatients and asymptomatic pyrexia would not have been noticed. Three patients in group 2 experienced mild rigors and symptoms of influenza in the first 24 hours after infusion. These symptoms did not recur with subsequent infusions.

\section{Discussion}

This study shows that pamidronate given at wide intervals over one year is as effective for the treatment of active Paget's disease as a short course of weekly treatment. Our findings also suggest that there is a dose-response relation with pamidronate. The more severely affected patients had a less marked reduction in the alkaline phosphatase activity in blood when given the same dose as less severely affected patients. Also, the six most severely affected patients in group $1 \mathrm{~A}$, who received a higher total dose of pamidronate than the remaining nine patients, subsequently had a better response. It may be that in more severely affected patients, who have a greater number of active pagetic lesions, the drug is spread more widely and therefore occurs at lower concentrations at the affected sites than in less severely affected patients. These observations are similar to those of Cantrill and Anderson ${ }^{19}$ who found

that the decrease in ALP activity was less marked where the initial ALP level was higher. In group 1 patients who received six weekly infusions, the ALP level continued to decrease after treatment was discontinued, and decreased at a similar rate to that observed in group 2 patients who received a single infusion every three months.

It is difficult to compare results between reported studies as Paget's disease may be of different severity in different groups. In addition, some patients might have been treated previously with bisphosphonates, which can remain in the skeleton for prolonged periods and can affect the response to further treatment. ${ }^{15}$ The decrease in ALP observed in this study, however, is similar to that noted by others with pamidronate given by mouth or intravenously. ${ }^{20}$

The optimum length of treatment is controversial. Some workers have found that short courses of daily or weekly treatment lead to a significant suppression of ALP that is still present one year after the start of treatment. ${ }^{19} 20$ In one study a single dose of $60 \mathrm{mg}$ of pamidronate was given intravenously once and this caused significant suppression of ALP to about $30 \%$ of the pretreatment level, which was still present after one year. All but one of these patients had mild disease and the one patient with severe disease responded poorly to this regimen. ${ }^{24}$

There are some features of this study which are interesting and unexpected. In a small number of patients the urinary excretion of hydroxyproline increased to greater than the pretreatment levels. In these patients the level of ALP in serum samples decreased. Although it is possible that this decrease may be caused by the relative inhibition of mineralisation caused by pamidronate, the concurrent decrease in the whole body retention of radiolabelled bisphosphonate suggests that it is more likely that bone turnover has been reduced and that these urine concentrations reflect the difficulty in obtaining true fasting samples from outpatients. This variability in hydroxyproline excretion has been noted previously in Paget's disease treated with pamidronate. ${ }^{25}$ The hydroxyproline to creatinine ratios began to increase after the end of treatment (group 1) or after three months (group 2). This is particularly surprising in group 2 as further treatment with pamidronate was being given to these patients. This may either be due to an 'escape' phenomenon, or it may be that there is a subgroup of osteoclasts that are relatively resistant to pamidronate.

Although there was a general reduction in tracer uptake in bone scans after treatment, this was not always seen and occasionally a lesion took up more of the radionuclide. This may also suggest that there are pagetic lesions present which are relatively resistant to treatment.

Although symptoms are notoriously difficult to assess, there appears to have been a significant decrease in pain and discomfort after treatment. Side effects occurred rarely and were self limiting, although these may preclude the initial dose being given to outpatients. ${ }^{26}$

In conclusion, pamidronate appears to be safe 
and effective in the treatment of Paget's disease. A dose-response effect exists, with more severely affected patients requiring a higher total dose to achieve disease suppression. In the short term an effective and convenient dosage regimen for patients with levels of ALP in serum samples up to four times the upper limit of the reference range is $45 \mathrm{mg}$ by intravenous infusion over four hours every three months for one year (i.e. a total dose of $180 \mathrm{mg}$ ). For those patients with ALP levels greater than this, larger doses may be more effective. Recommendations for the very long term remain conjectural.

We are grateful to Ciba-Geiby Pharmaceuticals Ltd for providing pamidronate for this study.

1 Francis M D, Russell R G G, Fleisch H. Diphosphonates inhibit formation of calcium phosphate crystals in vitro and pathological calcification in vivo. Science 1969; 165: $1264-6$.

2 Fleisch H, Russell R G G, Francis M D. Diphosphonates inhibit hydroxyapatite dissolution in vitro and bone resorption in vivo. Science 1969; 165: 1262-4

3 Smith R, Russell R G G, Bishop M. Diphosphonates and Paget's disease of bone. Lancet 1971; i: 945-7.

4 Ralston S H, Gardner M D, Dryburgh F J, Jenkins A S Cowan $\mathbf{R}$ A, Boyle I T. Comparison of aminohydroxypropylidene diphe IT. Comparison propylidene diphosphonate, mithramycin and corticocalcaemia. Lancet 1985; ii: $907-10$.

5 Yates A J P, Jones T H, Mundy K I, et al. Immobilisation Yates A J P, Jones T H, Mundy K I, et al. Immobilisation hypercalcaemia in adults

6 Tan T T, Alzaid A A, Sutcliffe N, Gardner M D, Thomson $\mathrm{J} A$, Boyle I T. Treatment of hypercalcaemia in thyrotoxicosis with aminohydroxypropylidene diphosphonate. Postgrad Med F 1988; 64: 224-7.

7 DeRose J, Singer F R, Avramides A, Flores A, Dziadiw R, Baker R K, Wallach S. Response of Paget's disease to porcine and salmon calcitonins. Am $\mathcal{J}$ Med 1974; 56: 858-66.

8 Ryan W G. Treatment of Paget's disease of bone with mithramycin. Clin Orthop 1977; 127: 106-10.

9 Woodhouse N J Y, Mohamedally S M, Saed-Nejad F, Martin $T$ J. Development and significance of antibodies to salmon calcitonin in patients with Paget's disease on long-term treatment. $B M 7$ 1977; ii: 927-9.

10 Mundy G R, Martin T J. The hypercalcaemia of malignancy pathogenesis and management. Metabolism 1982; 31 : 1247-77.

11 Heath D A. Treating Paget's disease. BMF 1987; 294: $1048-50$.

12 Boyce B F, Smith L, Fogelman I, Johnston E, Ralston S, Boyle I T. Focal osteomalacia due to low dose diphosphonate therapy in Paget's disease. Lancet 1984; i: 821-4.

13 Meunier P J, Chapuy M C, Alexandre C, et al. Effects of disodium dichloromethylene diphosphonate on Paget's disodium dichloromethylene diphospho

14 Delmas . P D, Chapuy M C, Vignon E, et al. Long term effects of dichloromethylene diphosphonate in Paget's disease of bone. F Clin Endocrinol Metab 1982; 54: 837-44.

15 Yates A J P, Percival R C, Gray R E S, et al. Intravenous clodronate in the treatment and re-treatment of Paget's disease of bone. Lancet 1985; i: 1474-7.

16 Frijlink $W$ B, Bijvoet $O$ L M, Velde J Te, Heynen G Treatment of Paget's disease with (3-amino-1-hydroxypropylidene)-1,1-bisphosphonate (APD). Lancet 1979; i $799-803$.

17 Fogelman I, Smith L, Mazess R, Wilson M A, Bevan J A. Absorption of oral diphosphonates in normal subjects. Clin Endocrinol 1986; 24: 57-62

18 Thiebaud D, Jaeger P H, Jacquet A F, Burkhardt P. Dose response in the treatment of hypercalcaemia by a single response in the treatment of hypercalcaemia by a
infusion of AHP-BP. $\mathcal{F}$ Clin Oncol 1988; 6: 762-8.

19 Cantrill J A, Buckler H M, Anderson D C. Low dose intravenous 3-amino-1-hydroxypropylidene-1,1-bisphosphonate (APD) for the treatment of Paget's disease of bone. Ann Rheum Dis 1986; 45: 1012-8.

20 Harinck H I J, Papapoulos S E, Blanksma H J, Moolenaa A J, Vermeij P, Bijvoet O L M. Paget's disease of bone: early and late responses to three different modes of treatment with aminohydroxypropylidene bisphosphonate (APD). BMF 1987; 295: 1301-5.

21 Cantrill J A, Anderson D C. Treatment of Paget's disease of bone. Clin Endocrinol 1990; 32: 507-18.

22 Gouerde B C, Veenkamp F J N. Routine assay of total urinary hydroxyproline based on resin-catalysed hydrolysis. urinary hydroxyproline based on

23 Smith M L. In: Fogelman I, ed. Bone scanning in clinical practice. Berlin: Springer, 1987: 244-6.

24 Thiebaud D, Jaeger P, Gobelet C, Jacquet A F, Burckhardt $P$. A single infusion of the bisphosphonate AHPrBP (APD) as treatment of Paget's disease of bone. Am $\mathcal{F}$ Med 1988; 85: 207-12.

25 Vega E, Gonzalez D, Ghiringhelli G, Mautalen C. Intravenous aminohydroxypropylidene bisphosphonate (APD) in the treatment of Paget's disease of bone. 7 Bone Miner Res 1987; 2: 267-71.

26 Gallacher S J, Ralston S H, Patel U, Boyle I T. Side effects of pamidronate. Lancet 1989; ii: 42-3. 